\title{
Erratum to: Trimethylamine- $N$-oxide: A Novel Biomarker for the Identification of Inflammatory Bowel Disease
}

\author{
Aze Wilson ${ }^{1,2,5}$ - Wendy A. Teft ${ }^{1} \cdot$ Bridget L. Morse $^{1} \cdot$ Yun-Hee Choi ${ }^{4}$. \\ Sarah Woolsey $^{5} \cdot$ Marianne K. DeGorter $^{1} \cdot$ Robert A. Hegele $^{3} \cdot$ Rommel G. Tirona $^{1,5}$. \\ Richard B. Kim ${ }^{1}$
}

Published online: 2 August 2015

(c) Springer Science+Business Media New York 2015

\section{Erratum to: Dig Dis Sci \\ DOI 10.1007/s10620-015-3797-3}

The original version of this article unfortunately contained an error in one of the coauthors' middle name. The author name was incorrectly published as Bridget $M$. Morse instead of Bridget L. Morse. This is corrected in the original article.

The online version of the original article can be found under doi:10.1007/s10620-015-3797-3.

Richard B. Kim

Richard.Kim@lhsc.on.ca

1 Division of Clinical Pharmacology, Department of Medicine, Western University, 339 Windermere Road B9-130, London, ON N6A 5A5, Canada

2 Division of Gastroenterology, Department of Medicine, Western University, 339 Windermere Road, London, ON N6A 5A5, Canada

3 Division of Endocrinology, Department of Medicine, Western University, 339 Windermere Road, London, ON N6A 5A5, Canada

4 Department of Epidemiology and Biostatistics, Western University, Kresge Building, Rm K201, London, ON N6A 5C1, Canada

5 Department of Physiology and Pharmacology, Western University, Medical Sciences Building, Rm 216, London, ON N6A 5C1, Canada 